\title{
The Future of Chemical Engineering: Did You Say The Triplet 'Processus- Product-Process' Engineering?
}

\author{
Jean-Claude Charpentier*
}

\begin{abstract}
One key to survival in globalization of trade and competition, including needs and challenges, is the ability of chemical engineering to cope with the society and economic problems encountered in the chemical and related process industries. It appears that the necessary progress will be achieved via a multidisciplinary and time and length multiscale integrated approach to satisfy both the market requirements for specific enduse properties and the environmental and society constraints of the industrial processes and the associated services. This concerns four main objectives for engineers and researchers: (a) total multiscale control of the process (or procedure) to increase selectivity and productivity, (b) design of novel equipment based on scientific principles and new methods of production: process intensification, (c) manufacturing end-use properties for product design: the triplet 'processus-product-process' engineering, (d) implementation of multiscale application of computational modeling and simulation to real-life situations: from the molecular scale to the overall complex production scale.
\end{abstract}

Keywords: Future of chemical engineering - Molecular modeling - Product design · Process intensification . Triplet 'processus-product-process' engineering

\section{Introduction}

The world moves forward. For the developing and industrializing countries, there is low cost of manpower and less constraining local production regulations. For the industrialized countries, there is a rapid development in consumer demand and constraints stemming from public concern over questions of environment and safety. In response to these changes, the world of chemistry and process industries such as petrochemical, pharmaceutical, agro and food, environment, surfactants, cosmetics and perfumes,

\footnotetext{
${ }^{*}$ Correspondence: Prof. Dr. Ing. Dir. J.C. Charpentier Department of Chemical Engineering/CNRS

Ecole Supérieure de Chimie Physique Electronique de Lyon

BP 2077 - 69616 Villeurbanne Cedex (France)

Tel.: +33472431702

Fax: +33472431670

E-Mail: charpentier@cpe.fr
}

electronics etc. is confronted, from the technological and scientific point of view, with a double challenge:

a) To research innovative processes for the production of commodity and intermediate products by no longer selecting processes solely on the basis of economic exploitation but by seeking compensating gains resulting from the increased selectivity and savings linked to the process itself. This requires value assessments of safety, health and environmental aspects, including the value of non-polluting technologies, reduction of raw material and energy losses and product and by-product recyclability.

b) To progress from traditional intermediate chemistry to new specialties and active material chemistry and parachemistry. This concerns industries involved with food products, with products for human, animal and plant health, with advanced materials, along with the chemistry/biology interface, i.e. the postgenomic world involving proteomics and metabolomics. This concerns upgrading and conversion of petroleum feed stocks and intermediates, conversion of coalderived chemicals or synthesis gas into fuels, hydrocarbons or oxygenates. The aim is characterized by new market objectives, with sales and competitiveness dominated by the end-use properties of a product linked to its quality or shape and size (i.e. chemical and biological stability, degradability, chemical, biological, and therapeutic activity, aptitude to dissolution, etc.). Control of end-use property and expertise in the design of the process, its permanent adjustments to variety, and changing demand along with speed in reacting to market conditions will be the dominant elements.

Being a key to survival in global markets including the previous needs and challenges, chemical and process engineering necessitate the current evolution in teaching and revolution in research. It involves the whole of scientific and technical knowledge necessary for physicochemical and biological transformations of raw material and energy into the targeted products required by the customer. But it is important to note that today $60 \%$ of all products that a chemical company sells to its clients are crystalline, polymeric or amorphous solids. These materials must have a clearly defined physical shape or texture in order to meet the designed and desired quality standards. 
This also applies to paste-like and emulsified products. Instead of classical basic and industrial chemicals, new developments increasingly concern highly targeted materials, active compounds, and special effect chemicals. These are much more complex in terms of molecular structure than classical chemicals.

\section{Le Génie du triplé 'Processus- Produit-Procédé': The Integrated Multidisciplinary and Multiscale Approach of Chemical and Process Engineering}

Thus chemical and process engineering is now concerned with the understanding and development of systematic procedures for the design and optimal operation of chemical and process systems, ranging from microsystems to industrial-scale continuous and batch processes, as presented in Fig. 1 by using the concept of chemical supply chain [1]. This chain starts with chemical and other products that industry must size and characterize at the molecule level. A subsequent step aggregates the molecules into clusters, particles, and thin films as single or multiphase systems that finally take the form of macroscopic mixtures - solids, paste-like or emulsion products. In the transition from chemistry or biology to engineering, one moves to the design and analysis of the production units, which are integrated into a process that in turn becomes part of an industrial site with multiple processes. Finally this site is a part of the commercial enterprise driven by market considerations and demands the inclusion of product quality. In this supply chain, it should be emphasized that product quality is determined at the micro and nano level and that a product with a desired property must be investigated for both structure and function. This involves a thorough understanding of the structure/property relationship at both molecular (e.g. surface physics and chemistry) and microscopic levels. And the ability to control microstructure formation to obtain the end-use properties of a fluid or solid product is the key to success and will help design and control product quality and make the leap from the nano level to the process level.

This necessitates an integrated system approach for a multiscale and multidisciplinary modeling of the complex, simultaneous and often coupled momentum, heat and mass transfer phenomena and processes taking place at different time scales $\left(10^{-15}-10^{8} \mathrm{~s}\right)$ and length scales $\left(10^{-8}-10^{6} \mathrm{~m}\right)$ encountered in industrial practice (Fig. 2) and involving approaches at the different length scales presented in Fig. 3.

So organizing scales and complexity levels in process engineering is now necessary to understand and to describe the relationships between events at nano and microscales to better convert molecules into useful products at the process scale. And organizing levels of complexity by translating molecular processes that I call processus into phenomenological macroscopic laws to create and to control the end-use properties and functionality of products manufactured by a continuous process underlie the current new views of chemical engineering. This can be de- fined by le Génie du triplé 'ProcessusProduits-Procédé' (triplet 'molecular Processes-Product-Process' engineering) with an integrated system approach of complex occurrences at different time and length scales. This explains why, in addition to the basic notions of unit operations, coupled transfers and classical tools of chemical engineering, that is, in addition to the fundamentals of chemical engineering (separation engineering, chemical reaction engineering, catalysis, transport phenomena, optimization, and process control), this integrated multidisciplinary and multiscale approach is a supplementary and considerable advantage for the development and the success of this engineering science in terms of concept and paradigms. It is of a great help in order to analyze, design, and operate processes able to manufacture a product - first on the market - (often having a short life cycle) with the desired property and optimally thanks to processes involving if possible zero defect, zero pollution and zero accident.

And it will be possible to understand and to describe the relationship between events at different scales thanks to the large breakthroughs (a) in molecular modeling (both theory and computer simulation), (b) in scientific instrumentation and non-invasive measurement techniques (NMR, TAP, tomographic techniques, spectroscopic or monochromatic ellipsometry, i.e. diffusing wave spectroscopy, etc.), and related nanotechnologies in connection with image processing, and (c) in powerful computational tools and capabilities, necessary for the treatment of generalized local informa-

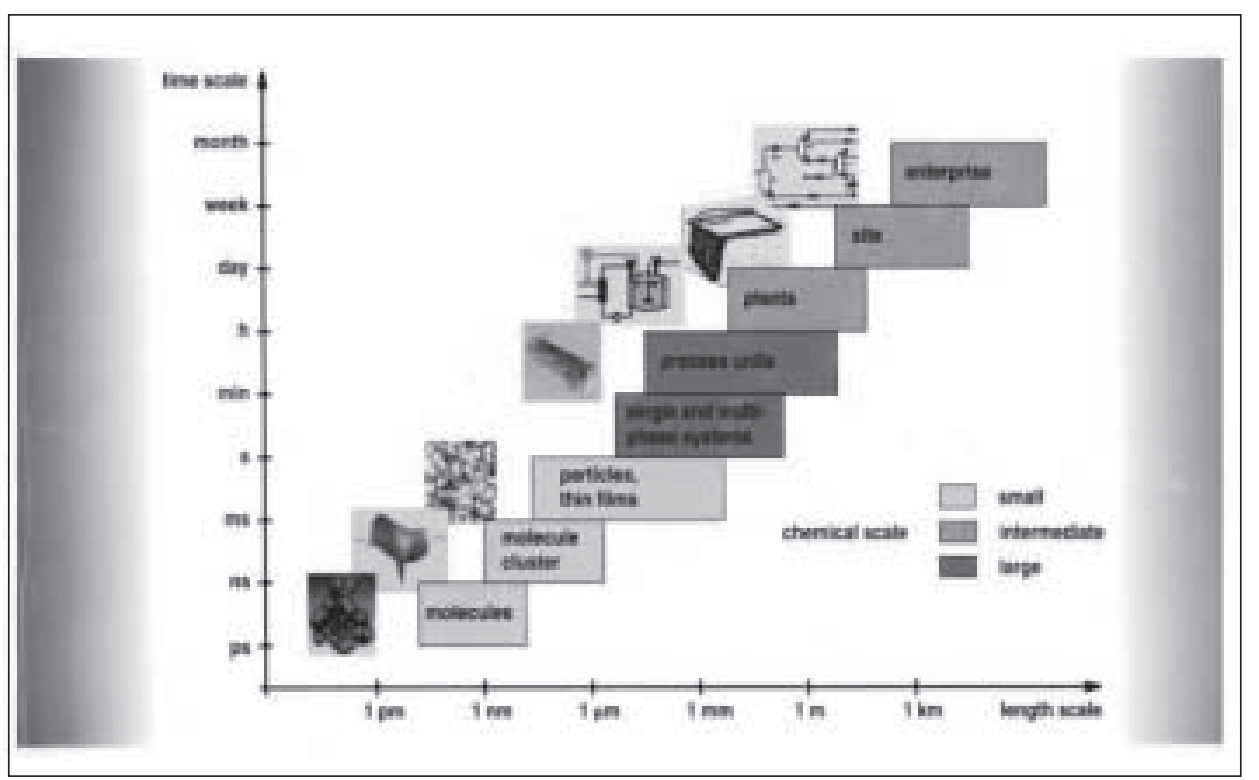

Fig. 1. Chemical supply chain (Grossmann and Westerberg, 2000 [1]) 

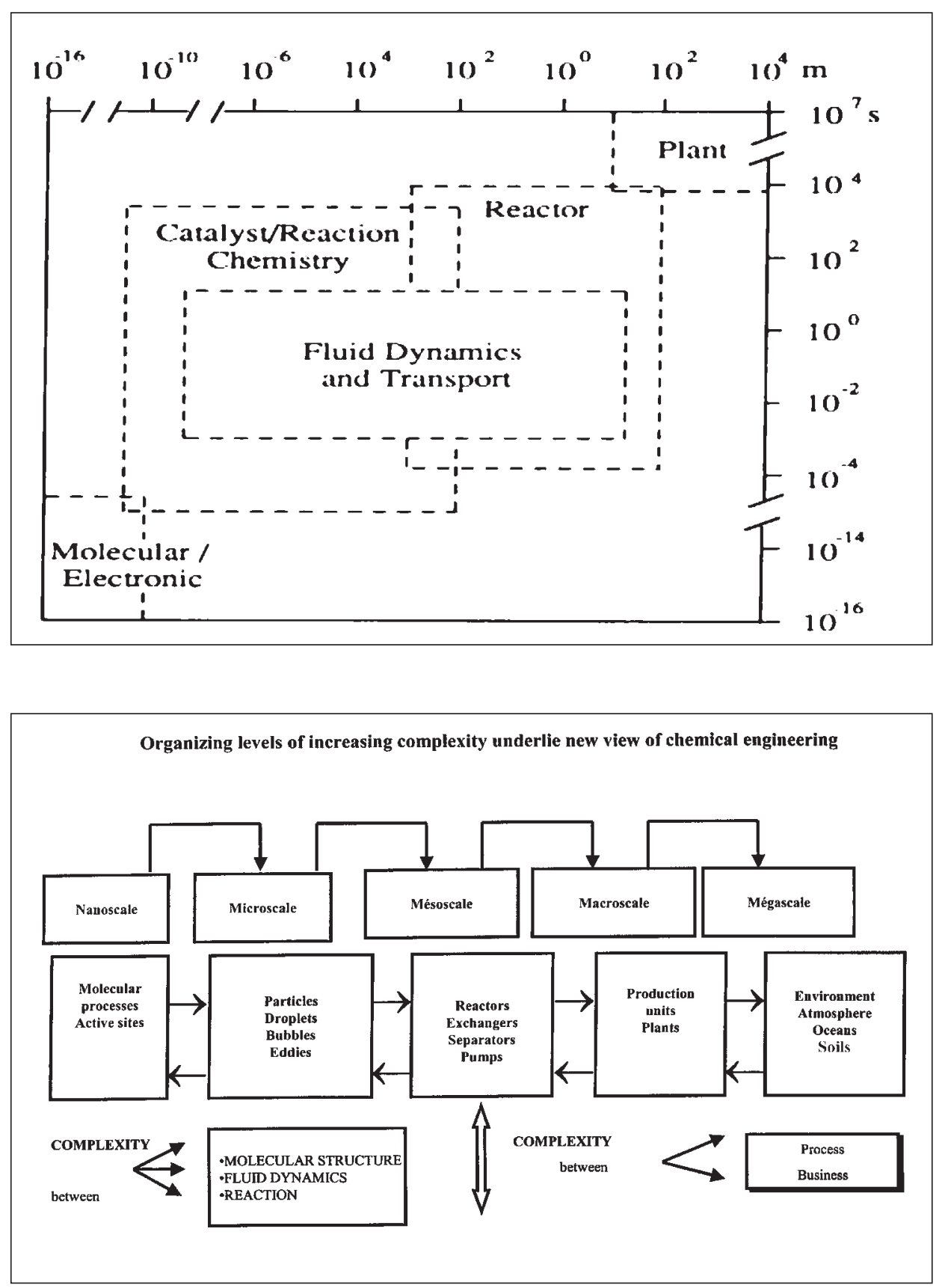

tion (increasing use of computational fluid dynamic or mixing, e.g. CFDLIB, FLUENT, PHOENICS, FLOW 3D, FIDAP, FLOWMAP, etc.) [2].

\section{Chemical and Process \\ Engineering: Quo vadis? Four Main Objectives}

\subsection{Total Multiscale Control of the Process to Increase Selectivity and Productivity}

This necessitates the 'intensification' of operations and the use of precise nano and micro technology design. Such is the case of molecular information engineering encountered for supported organo-
Fig. 2. The length and time scales covered in the multiscale approach.
Fig. 3. Scales and complexity levels in process engineering: to understand and to describe the relationships between events at nano- and microscales to better convert molecules onto useful products on the process scale.

ty by supplying the process with a local 'informed' flux of energy or materials is another opportunity. Indeed at the higher microscale level, detailed local temperature and composition control through staged feed and heat supply or removal would result in higher selectivity and productivity than the conventional approach, which imposes boundary conditions and lets a system operate under spontaneous reaction and transfer processes. Finding some means to convey energy to the site (supplying the process with a local 'informed' flux of energy) where it may be utilized in an intelligent way is therefore a challenge.

More clearly recognized is the necessity to increase information transfer in the reverse direction, from process to man: developing all kinds of intelligent sensors, visualization techniques, image analysis, and on-line probes giving instantaneous and local information about the process state. This opens the way to a 'smart chemical and process engineering' requiring close computer control, relevant models, and arrays of local sensors and actuators. 


\subsection{Design of Novel Equipment}

Based on Scientific Principles and New Operating Modes and Methods of Production: Process

\section{Intensification}

The progress of basic research in chemical engineering has led to a better understanding of elementary phenomena and now makes it possible to imagine new operating modes of equipment or to design novel equipment based on scientific principles. Such is the case with the 'multifunctional' equipment that couple or uncouple elementary processes (transfer - reaction - separation) to increase productivity, selectivity with the desired product or to facilitate the separation of undesired by-products. In recent years, extractive reaction processes involving single units that combine reaction and separation operations have received considerable attention as they offer major advantages over conventional processes: due to the interaction of reaction and mass and energy transfer, thermodynamic limitations, such as azeotrope, may be overcome and the yield of reactions increased. So the reduction in the number of equipment units leads to reduced investment costs and significant energy recovery or savings. Furthermore improved product selectivity leads to a reduction in raw material consumption and, hence, operating costs. So globally, process intensification through use of multifunctional reactors permits significant reductions in both investment and plant operating costs (10-20\% reductions) by optimizing the process. In an era of emaciated profit margins, it allows chemical producers more leverage in competing in the global market place.

There exists a great number of reactive separation processes involving unit operation hybridization. The concept of reactive or catalytic distillation has been successfully commercialized, both in petroleum processing, where packed bed catalytic distillation columns are used, and in manufacture of chemicals where reactive distillation is often employed. Catalytic distillation combines reaction and distillation in one vessel using structured catalysts as the enabling element. An alternative reaction-separation unit is the chromatographic reactor, which utilizes differences in adsorptivity of the different components involved rather than differences in their volatility. It is especially interesting as an alternative to reactive distillation when the species involved exhibit small volatility differences or are either non-volatile and sensitive to temperature, as in the case, for exam- ple, in small fine chemical or pharmaceutical applications. Typical examples for the adsorbents used are activated carbon, zeolites, alumina, ion-exchange resins, and immobilized enzymes [4]. Concerning the coupling of reaction and crystallization, there exist myriads of basic chemical, pharmaceuticals, agricultural products, ceramic powders, and pigments produced by reactive crystallization-based processes. These separation processes are synthesized by bypassing the thermodynamics barriers imposed on the system by the chemical reactions and the solubilities of the components in the mixture. By combining crystallizers with other unit operations, the stream compositions can be driven to regions within composition space where selective crystallization can occur [5a]. The complementary nature of crystallization and distillation is also explored. Indeed the hybrids provide a route to bypass thermodynamic barriers in composition space that neither distillation, which is blocked by azeotropes and hindered by tangent-pinches in vaporliquid composition space, nor selective crystallization, which is prevented by eutectics and hampered by solid solutions and temperature-insensitive solubility surfaces, can overcome when used separately [5b].

Membrane technologies respond efficiently to the requirement of so-called process intensification because they allow improvements in manufacturing and processing, substantially decreasing the equipment-size/production-capacity ratio, energy consumption, and/or waste production and resulting in cheaper, sustainable technical solutions. The paper by Drioli and Romano [6] documents the state of the art with respect to progress and perspectives. This technology can respond to the strongly increasing demand for food additives, feeds, flavors, fragrances, pharmaceuticals, agrochemicals, etc.

Finally, though multifunctional reactors are not quite new to the process industries, i.e. absorption or extraction with chemical reaction, only recently reactors incorporating several 'functions' in one reactor have been formally classified as being multifunctional [7] and the large benefits obtained in integrating progress of knowledge at different scale and timelengths have been acknowledged by the process industries. However we will mention that the use of hybrid technologies encountered with multifunctional reactors is often limited by the resulting problems concerning control and simulation.
The intensification of processes may be obtained by new modes of production which are also based on scientific principles: reversed flow for reaction-regeneration, countercurrent flow and induced pulsing flow in trickle beds, unsteady operations, cyclic processes, extreme conditions, etc. Current production modes are currently and will in future be more and more challenged by decentralization, modularization, and miniaturization. Indeed microtechnologies recently developed lead to microreactors, micromixers, microseparators, micro-heat-exchangers, and microanalyzers, making possible accurate control of reaction conditions with respect to mixing, quenching, and temperature profile.

Miniaturization of chemical analytic devices in micro-total-analysis-system ( $\mu \mathrm{TAS}$ ) represents a natural extension of microfabrication technology to biology and chemistry with clear applications in combinatorial chemistry, high-throughput screening, and portable analytical measurement devices. Also the merging of $\mu$ TAS techniques with microreaction technology promises to yield a wide range of novel devices for reaction kinetic and micromechanism studies, and online monitoring of production systems [8].

Moreover scale-up to production by replication of microreactors units used in the laboratory would eliminate costly redesign and pilot plant experiments, thereby shortening the development time from laboratory to commercial-scale production. This approach would be particularly advantageous for pharmaceutical and fine chemicals industries where production amounts are often less than a few metric tons per year.

For illustration recently de Bellefon $e t$ al. [9] proposed a new concept for highthroughput screening (HTS) experiments for rapid catalyst screening based on dynamic sequential operations with a combination of pulse injections and micromachined elements. They describe a new concept to achieve HTS of polyphasic fluid reactions for two test reactions, a liquid-liquid isomerization of allylic alcohols and a gas-liquid asymmetric hydrogenation. The principle used for the test microreactor is a combination of pulse injections of the catalyst and the substrate, a IMM micromixer with negligible volume and residence time less than $10^{-2} \mathrm{~s}$, and a tubular reactor (Fig. 4). The two scanning electron microscopy images show the micromixer, with $2 \times 15$ interdigitated microchannels $(25 \mu \mathrm{m}$ width $)$ with corrugated walls. The pulses mix 
perfectly in the micromixer and the liquids or gas-liquid mixtures thereby emulsify and behave as a reacting segment, which then travels along the tubular microreactor. Collection at the outlet of the reactor and analysis afford the conversion and selectivity data. The results led to the selection of the best catalyst showing activity towards a large class of allylic alcohols. Similar results which were obtained in a microreactor and in a traditional well-mixed batch reactor $\left(40 \mathrm{~cm}^{3}\right)$ prove the validity of the concept (testing frequencies of more than 500 p.d). Using these microreactors for dynamic, high-throughput screening of fluid-liquid molecular catalysis offer considerable advantages over traditional parallel batch operations: ensuring good mass and heat transport in a small volume, reduced sample amounts (to $\mu \mathrm{g}$ levels), larger range of operating conditions and simpler electro-mechanical moving parts.

\subsection{Formulation or Product Design: Manufacturing End-use Properties}

This is the answer for the current ever-growing market place demand for sophisticated products combining several functions and properties: cosmetics, detergents, surfactants lubricants, textiles, inks, paints, rubber, plastic composites, pharmaceuticals, drugs, foods, agrochemicals, and more. This product engineering (synthesis of properties), as also previously seen, is the translation of molecular structure into macroscopic phenomenological laws in terms of state variables. In practice it mostly concerns complex media and the so-called 'soft solids' systems which have a detectable yield stress, such as ceramics, pastes, foods or drilling muds. Product design concerns also particulate solids encountered in $70 \%$ of the process industries. This involves the creation and the control of the particle size distribution in operations such as crystallization, precipitation, prilling, as well as the control of the particle morphology and the final shaping and presentation in operations such as agglomeration, calcination, compaction, encapsulation. Both types of operations need a better understanding as they control the end-use property and quality features, such as taste, feel, smell, color, handling properties, sinterability or biocompatibility.

The quality and properties of emulsified or paste-like and solid products is determined at the micro and nano level. Therefore to be able to design and control the product quality and make the leap from the nano level to the process level,

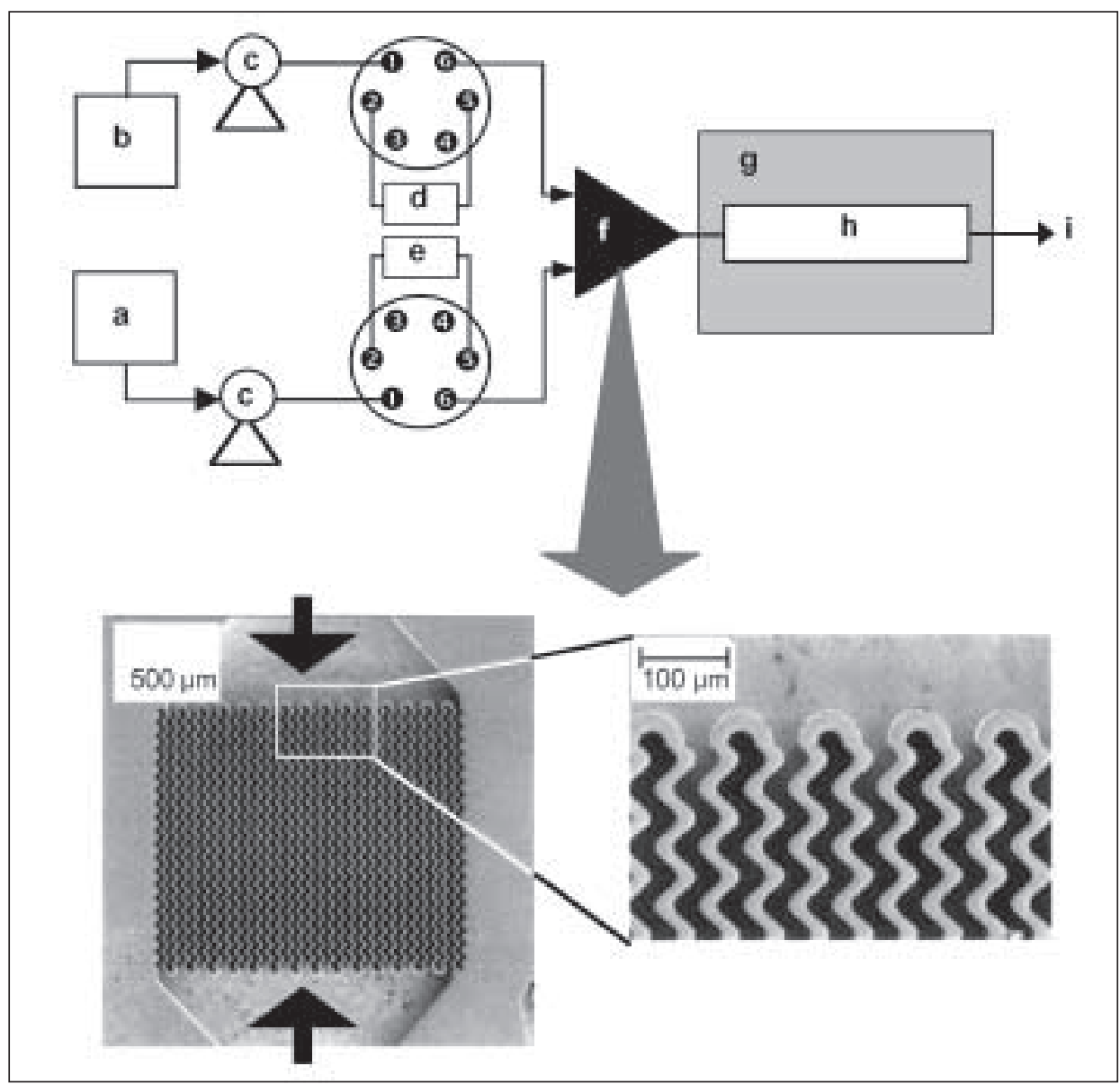

Fig. 4. The IMM micromixer for high-throughput screening: the two scanning electron microscopy images show the $2 \times 15$ interdigitated microchannels with corrugated walls (de Bellefon et al. [9]). Experimental set-up: a water reservoir, $\mathbf{b} n$-heptane reservoir, $\mathbf{c}$ high pressure liquid pumps, d HPLC type injection valve equipped with a $200 \mu$ loop, e HPLC injection valve with a $1 \mathrm{ml}$ loop, f micromixer, $\mathbf{g}$ thermoregulated bath, $\mathbf{h}$ tubular stainless steel reactor $(0.4 \mathrm{~cm}$ i.d., $80 \mathrm{~cm}$ length), i outlet to analytics, j SEM image of the mixing microelement showing the $2 \times 15$ interdigitated microchannels ( $25 \mu \mathrm{m}$ width) with corrugated walls.

chemical and process engineering involved with structured materials have to face many challenges in fundamentals, in process integration, in simulation, and in process control. For illustration we may cite the control of the shape and size of crystals in an industrial crystallization process. Wintermantel [10] has shown that much improved process control, both in terms of crystal purity and a defined size distribution, could result by detailed computer studies of the crystallization processus which can be remarkably changed by the presence of small traces of foreign substances such as unwanted by-products in the feed solution. Indeed in order to understand the mechanisms causing these changes in crystal size and shape so that it is possible to utilize them in a controlled manner, one must explain the structure-activity relationships on a molecular level. And with computer simulations, diagrams of the molecular struc- ture of the most important crystal surfaces can be generated from X-ray crystal structure data. Also in the same computer simulations, contaminant molecules or molecules with an expected beneficial effect on crystallization processus can be placed on each crystal surface and their adsorption energy calculated. If the hypothesis is that the growth rate of surface decreases with increasing adsorption energy, comparison of the relative adsorption energies could lead to the prediction of the expected modified crystal shape. This was illustrated with the results of crystallization from a feed ammonium sulphate solution containing the dye amaranth. It was shown that the molecule amaranth is adsorbed onto the 001 surface of ammonium sulphate crystals with the highest adsorption energy in comparison with the other crystal surface. And according to the calculations, the somewhat block-shaped crystal produced in 
the pure system becomes a flat shaped crystal having a large 001 surface area, which was experimentally verified.

Another illustration of this multilevel research effort in crystallization has been recently proposed in the area of electrical engineering: microelectronics. It concerns correlations between operating conditions and microstructure of Low Pressure Chemical Vapor Deposit (LPCVD) silicon-based films prepared from silane $\mathrm{SiH}_{4}$. The main aim is the development of a rigorous simulation model for the interpretation of the layer growth experimental data by taking into account both the mass transfer resistance at boundary layer and the solid layer growth kinetic expression (Dollet et al. [11]). The four blocks of accessible knowledge for a LPCVD process are presented in Fig. 5. Specialists in material sciences may relate microstructure and properties of thin films while specialists in chemical engineering can correlate and simulate the macroscopic operating conditions of a CVD reactor and the local conditions inside the reactor. It is clear that any relationship established between blocks 2 and 3 affords an intrinsic property of the analyzed system which is independent of the equipment used and which allows to treat the complete design or optimization problems completely by

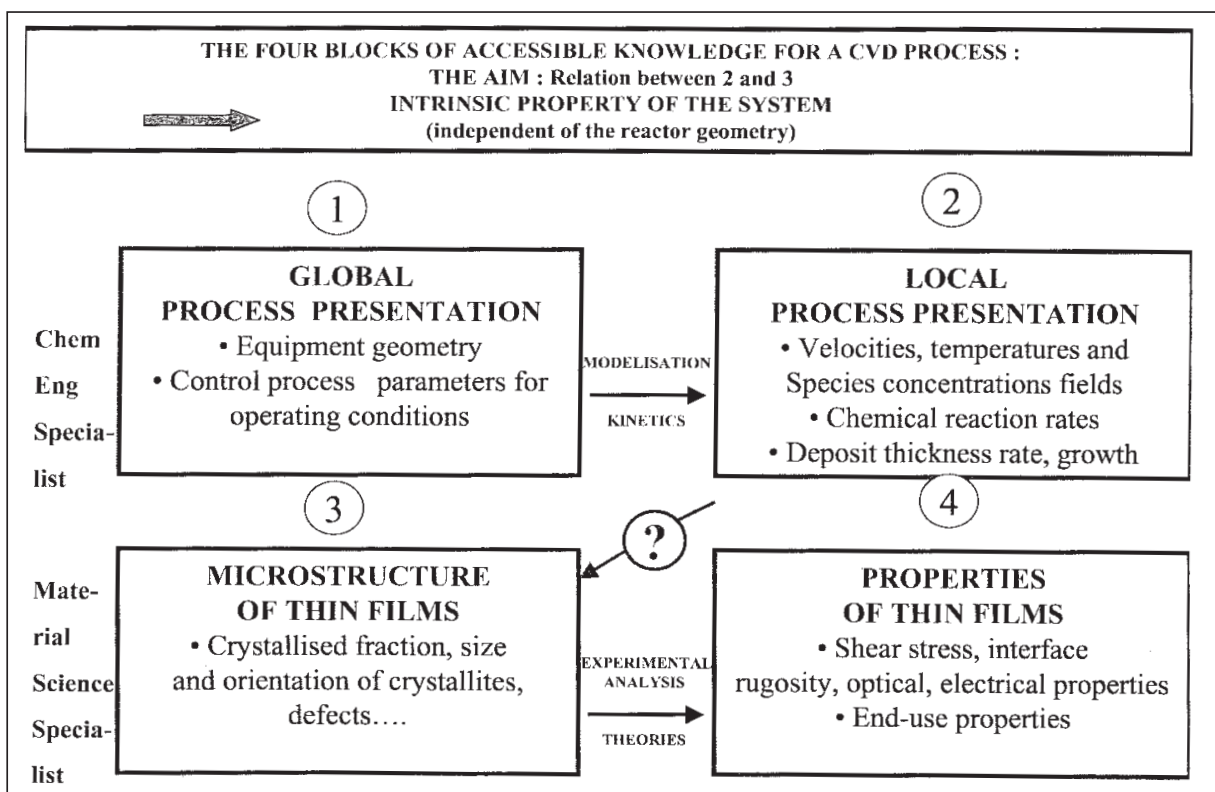

Fig. 5. The four blocks of accessible knowledge for a CVD process. The aim: the relation between 2 and 3 is an intrinsic property of the system independent of the reactor geometry. theoretical methods. The thin CVD layers were produced in the so-called hot wall tubular reactor ( $2 \mathrm{~m}$ length, 2.2 in diameter). Numerous characterization methods have been used by the authors to study the microstructural evolution of the layers in function of their elaboration conditions. The local state of the reactor was simulated by a model CVD includ-

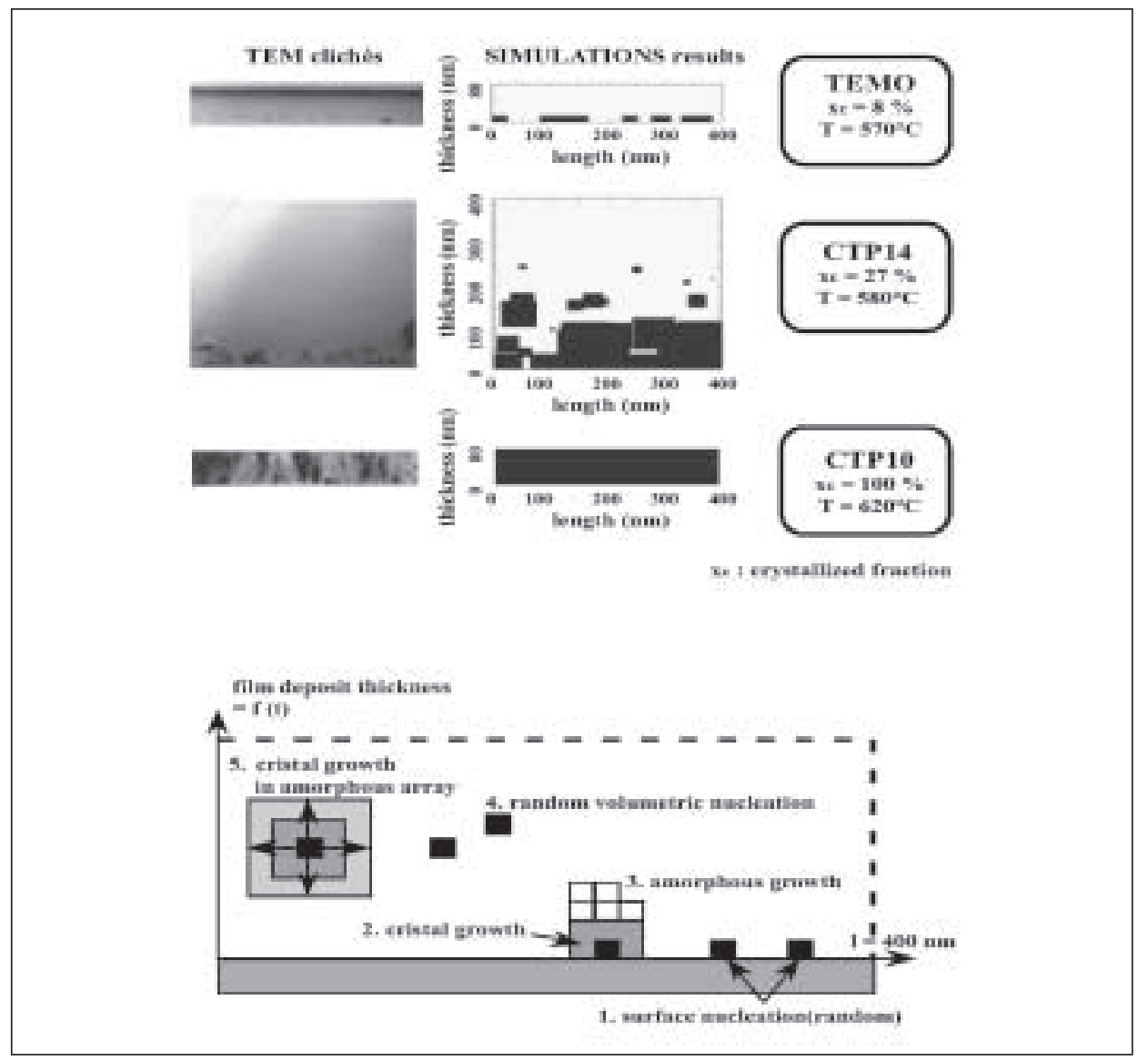

ing the gaseous flow hydrodynamic, mass transfer and chemical reaction parameters both in heterogeneous phase (gas-solid interface) and in homogeneous phase (solid phase). Mechanisms existing at the surface substrate and nucleation and crystal growth phenomena together with the dynamic characteristics of the microstructure formation were modeled with a simple geometric and statistic approach based on concepts of the mechanics of continuous media (instead of using molecular dynamic models requiring too much computer power). The final model that necessitates the local conditions of elaborations at surface substrate and nucleation and crystal growth laws, is able to calculate the thickness, the fraction of crystallized silicon and the space distribution of silicon crystallites in the deposit layer films from operating conditions such as gas phase components, temperature, pressure, and initial substrate nature or surface state. A comparison between the TEM micrographies of the experimental silicon films from pure silane for different temperatures and deposit durations and the simulation of the

Fig. 6. Microstructure of silicon LPCVD films from silane at different temperatures: (left) TEM cliches, (right) simulation results (Dollet et al. 2000 [11]). 
model has shown a good approximation for the crystallized fraction $x_{c}$ and the space distribution of crystallites (Fig. 6).

Finally, much progress has been realized over the past few years for the product design and the control of the process using the scientific methods of chemical engineering: thermodynamic equilibrium states are examined, transport processes and kinetics are analyzed separately, and these are linked again by means of models with or without the help of molecular simulation and finally with the help of computer tools for simulation, modeling and extrapolation at different scales for the whole supply chain (BASF, Unilever, Degussa, Astra Zeneca, Nestlé, etc.). But how can operations be scaled up from laboratory to plant? Will the same product be obtained and will its properties be preserved? What is the role of equipment design in determining product properties? This leads to the fourth main objective.

\subsection{Implementation of Multiscale and Multidisciplinary Computational Chemical Engineering Modeling and Simulation to Real-life Situations: From the Molecule to the Overall Complex Production Scale into the Entire Production Site}

Computers have opened the way for chemical engineering in the modeling of molecular and physical properties on the nano and microscopic scales. For the molecular modeling, application of the principles of statistical molecular mechanics computational techniques (Monte-Carlo and molecular dynamics) and quantum mechanics constitute an area for the problem-oriented approach of chemical engineering. Molecular modeling starts from a consideration of microscopic structure and molecular interactions in a material system and derives thermodynamic, transport, rheological, mechanical, electronic properties through rigorous deductive reasoning based on the principles of quantum and statistical mechanics.

Furthermore, turning to the macroscopic scale, dynamic process modeling and process synthesis are also being increasingly developed. Indeed, one must remember that the targeted products in question are generally not mass-produced products but ones which are produced in small batches and just in time for delivery to the consumer. And to be competitive under these conditions, it is particularly important to analyze and optimize the supply chains for which we are interested in the time that the individual process steps take, and these have to be simulated and evaluated also in terms of costs. But in chemical and parachemical processes, the location of a particular component in the supply chain at a given time is not well defined. Indeed a batch can be found in a stirred tank, a filter, a dryer, a pump, a mill, and a storage container simultaneously. New event-driven simulation tools help solve these problems by simulating both material flows and states within the individual pieces of equipment. This dynamic simulation may make it possible to see in a matter of seconds whether bottle-necks may occur in the plant over the course of days, months or years. Moreover the ability to use a series of coherent tools (i.e. software) during the process life cycle is now well established: integration and opening of modeling and event-driven simulations environments are these days taking on a more and more important role, see for example the Computer Aided Process Engineering European Brite Euram program CAPE-OPEN. The aim is to promote the adoption of a standard of communication between simulation systems at any time- and length-scale level (thermodynamic, unit operations, numerical utilities for dynamic, static, batch simulations, fluid dynamics, process synthesis, energetics integration, control process) in order to simulate processes allowing the customers to integrate information from any simulator onto another one. For the future, the Global CAPE-OPEN (GCO) project is expanding and developing interface specification standards to insure interoperability of CAPE software components. And a standardization body (CAPE-OPEN Laboratories Network, $\mathrm{CO}-\mathrm{LaN}$ ) has been established to maintain and disseminate the software standards in the CAPE domain and to ensure that software tools reach a level of interoperability that will help ensure sustained growth and competitiveness [12].

\section{Conclusions. Chemical and Process Engineering in the Future: A Multidisciplinary Key Technology Serving Mankind and Sustainable Technologies}

The goals of a transition to sustainability are promoted by the current scientifically relevant multidisciplinary and interdisciplinary integrated approach of chemical engineering at any time- and length-scales from the molecular and micro-scales processes up to the plant-scale process to design and produce the desired products at the mesoscale with environmental, societal and economics requirements. And in the future within the framework of the four cited objectives, chemical engineering will be more concerned and involved with the application of Life Cycle Assessment to the triplet 'processus-product-process', i.e. application of LCA not only to product design and its use but also to the plant and equipment together with the associated services.

Received: February 19, 2002

[1] I.E. Grossmann, A.E. Westerberg, AIChE Journal 2000, 46, 1700

[2] J.C. Charpentier, 'The world of Chemistry and Process industries at the heart of a great number of Scientific and Technological challenges: in a context of 'market demands versus technological offers' is the new chemical engineering able to help and why?', 3rd European Congress in Chemical Engineering, invited plenary lecture, Nürnberg, 26-28 June 2001.

[3] J. Ying, AIChE Journal 2000, 46, 1902.

[4] D. Lode, M. Houmard, C. Migliorini, M. Mazotti, M. Morbidelli, Chem. Eng. Science 2001, 56, 269.

[5] a) D.A. Berry, K.M. Ng, AIChE Journal 1997, 43, 1737; b) D.A. Berry, K.M. Ng, AIChE Journal 1997, 43, 1751.

[6] E. Drioli, M. Romano, Ind. Eng. Chem. Res. 2001, 40, 1277.

[7] F.M. Dautzenberg, M. Mukherjee, Chem. Eng. Science 2001, 56, 251.

[8] K.F. Jensen, Chem. Eng. Science 2001, 56, 293.

[9] C. de Bellefon, N. Tanchoux, S. Caravieilhes, P. Grenouillet, V. Hessel, Angew. Chem. Int. Ed. 2000, 39, 3442.

[10] K. Wintermantel, Chem. Eng. Science 1999, 54, 1601.

[11] A. Dollet, B. Caussat, J.P. Couderc, B. de Mauduit, Entropie 2000, 226, 3.

[12] M. Pons, B. Braunschweig, K. Irons, J. Köller, A. Kuckelberg, P. Roux, 'CAPE OPEN standards: implementation and maintenance', 3rd European Congress in Chemical Engineering, Computer Engineering Session 7, Poster 188, Nürnberg 26-28 June, 2001. 